\title{
dynamical structural science
}

Acta Crystallographica Section A

Foundations of Crystallography

ISSN 0108-7673

\section{Dynamical structural science}

\author{
E. Collet \\ Institut de Physique de Rennes, Université de Rennes1-CNRS, UMR 6251, 35042 Rennes, France, \\ and Institut Universitaire de France, 103 bd Saint-Michel, 75005 Paris, France
}

Structural science has played a key role in understanding the microscopic origin of physical properties of matter at thermal equilibrium and in studying the response of matter to changes of external parameters such as pressure and temperature. In the recent past, a new challenge has emerged: to direct the functionality of matter by light excitation on the relevant timescale. Advances in ultra-short laser technology opened the way for driving and tracking ultra-fast transformations of molecules in solution and in the gas phase: the field of femtochemistry was born and its importance was recognized by the Nobel Prize in chemistry awarded to Ahmed H. Zewail, who has contributed an introductory perspective to this issue. In parallel, the field of crystallography has been enriched by investigations of photoexcited crystals, in which the constitutent molecules transform under continuous or pulsed light irradiation. This is the field of photocrystallography.

Recent advances in pulsed electron and X-ray sources now make it possible to track structural reorganization in real time. As the pulse length can today be as short as $100 \mathrm{fs}$ ( $1 \mathrm{fs}=10^{-15} \mathrm{~s}$ ), optical pump coupled to X-ray or electron probe experiments allow tracking of the atomic or molecular motions on their intrinsic elementary timescale, i.e. the phonon frequency. As these techniques are now well established, the time is ripe to expose them to the broadest community of crystallography by a special issue of Acta Crystallographica Section A: Foundations of Crystallography, dealing with dynamical structural science. The issue was prepared with the assistance of Philip Coppens and Dieter Schwarzenbach.

Recent years have seen terrific successes in providing atomic level views of transient phenomena in inorganic, molecular and even biomolecular systems. This issue clearly shows that the field of dynamical structural science, illustrated by selected contributions, is truly interdisciplinary in nature. A large variety of topics is presented, dealing with solid-state physics (D. Miller et al., S. Johnson et al., T. Elsaesser et al.), molecular materials (P. Coppens et al., H. Cailleau et al.), biological molecules (M. Schmidt et al., R. Neutze et al., S. Adachi et al.) and molecules in solution (M. Chergui, L. Chen et al., M. Wulff et al., M. Nielsen et al., H. Ihee et al.). The timescales of interest also span a broad range, from $100 \mathrm{fs}$ atomic motion dynamics to slower $(\mu \mathrm{s})$ molecular diffusion and ms relaxation processes of transient excited states. Finally, the different natures of the probes (X-ray and electron) and of the techniques (spectroscopy and scattering/ diffraction) lead to complementary signals, which can be combined in comprehensive studies. The connection with state-of-the-art data analysis as well as quantum chemistry theory makes it possible to elucidate transient states of matter hidden, and sometimes coexisting, in the time domain.

These landmark studies were enabled by breakthroughs in probing with high time resolution using X-ray and electron pulses. Mechanical choppers can generate $\mu$ s pulses and are used around intense third-generation synchrotron sources. Choppers may also select a single X-ray pulse from a synchrotron ring. Synchronization with a femtosecond laser makes it possible to reach $100 \mathrm{ps}$ (1 ps = $10^{-12} \mathrm{~s}$ ) time resolution, limited currently by the X-ray pulse duration. A femto- 


\section{dynamical structural science}

second laser may be used for slicing an electron bunch inside a synchrotron ring, generating $100 \mathrm{fs} \mathrm{X}$-ray pulses. Alternative solutions for generating $100 \mathrm{fs} \mathrm{X}$-ray pulses also exist with laser-driven plasma X-ray sources. This time resolution can also be reached with electrons, thanks to the design of new electron guns. Finally, the new generation of incredibly bright $\mathrm{X}$-ray free electron lasers (X-FELs) will open new possibilities for developing the multidisciplinary field of dynamical structural science.
Thanks to the different contributors acknowledged here, preparing this special issue was a unique opportunity to present a broad spectrum of recent advances in this field, which set the stage for imminent breakthroughs in the understanding of our dynamical world. A new era of adventure is now offered to our community. To paraphrase Stanley Kubrick, let us summarise the future by '2010: Beginning of a New Reciprocal Space Odyssey'. 